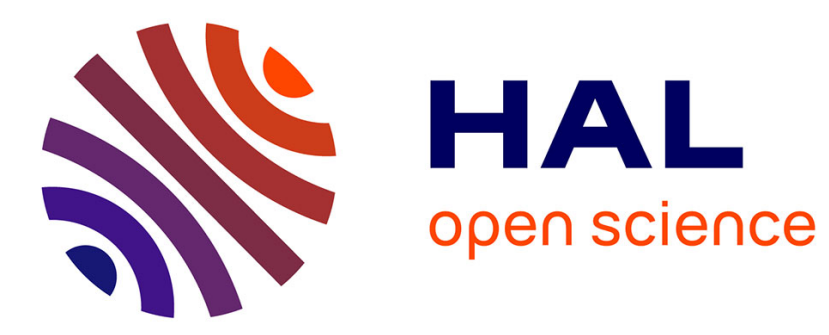

\title{
Une généalogie de la pensée managériale sur la RSE
} Aurélien Acquier, Franck Aggeri

\section{To cite this version:}

Aurélien Acquier, Franck Aggeri. Une généalogie de la pensée managériale sur la RSE. Revue Française de Gestion, 2008, 1 (180), pp.131-157. halshs-00645679

\section{HAL Id: halshs-00645679 \\ https://shs.hal.science/halshs-00645679}

Submitted on 25 Jan 2016

HAL is a multi-disciplinary open access archive for the deposit and dissemination of scientific research documents, whether they are published or not. The documents may come from teaching and research institutions in France or abroad, or from public or private research centers.
L'archive ouverte pluridisciplinaire HAL, est destinée au dépôt et à la diffusion de documents scientifiques de niveau recherche, publiés ou non, émanant des établissements d'enseignement et de recherche français ou étrangers, des laboratoires publics ou privés. 
Une g\&eacute;n\&eacute;alogie de la pens\&eacute;e manag\&eacute;riale sur la RSE

Acquier, Aurélien;Aggeri, Franck

Revue Française de Gestion; Jan 2008; 34, 180; ProQuest pg. 131

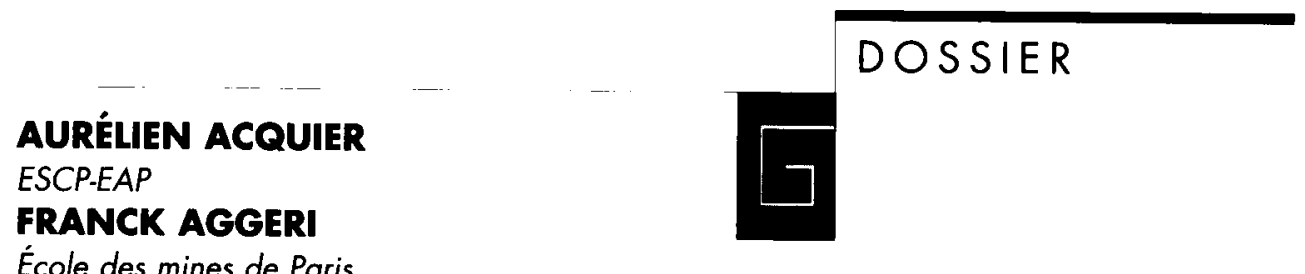

École des mines de Paris

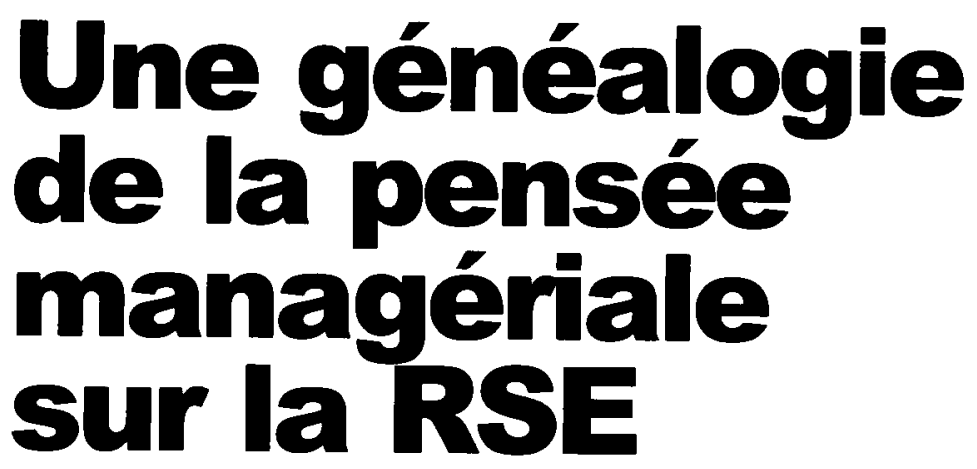

Cet article propose une revue de littérature généalogique des concepts gestionnaires associés à la responsabilité sociale de l'entreprise (RSE). Nous analysons le développement historique du concept de RSE en Amérique du Nord, et réévaluons la portée des concepts et des cadres d'analyse gestionnaires associés à ce construit (approches responsiveness, performance sociétale de l'entreprise, approches par les parties prenantes, etc.) Ce travail débouche sur une typologie des principaux courants de recherche en matière de RSE. 
$\mathrm{L}$ e renouvellement rapide des concepts et des théories est l'une des caractéristiques des sciences de gestion. Souvent issues de l'observation des pratiques managériales, les théories cherchent à éclairer les actions contemporaines des entreprises au risque d'alimenter la critique de participer à la formation des “ modes managériales " du moment (Abrahamson, 1991). Cette critique concerne notamment le domaine de la responsabilité sociale de l'entreprise (RSE), ensemble de pratiques et de théories en interactions étroites, qui connaît un succès croissant depuis une dizaine d'années dans le monde socio-économique et dans le monde académique. L'intérêt des acteurs économiques et sociaux (entreprises, pouvoirs publics, ONG, etc.) se manifeste, entre autres, par une intense production de discours (exemple: rapports de développement durable), de nouvelles fonctions managériales, d'outils et normes en matière reporting extra-financier. Sur le plan académique, l'intérêt des chercheurs pour la RSE se manifeste par un foisonnement conceptuel (exemple: RSE, responsiveness, stakeholder, performance sociale de l'entreprise, corporate citizenship, etc.) et par une forte hétérogénéité des travaux en tous genres (empiriques, normatifs, économétriques, etc.), parmi lesquels il est parfois difficile de trier le bon grain de l'ivraie.

Le projet de cet article est de considérer ces concepts et ces théories selon une approche généalogique $^{1}$, c'est-à-dire en analysant leurs filiations et en les replaçant dans les débats théoriques, institutionnels et pratiques dans lesquels ils ont été conçus et ont été diffusés. Une revue de littérature traditionnelle tendrait à identifier et confronter, à un moment donné, différentes écoles de pensées en concurrence en matière de RSE (Garriga et Melé, 2004), en analysant leur portée explicative à l'aune de leurs postures ontologiques et épistémologiques respectives (Burrell et Morgan, 1979). La démarche généalogique vise, à l'inverse, à comprendre la manière dont différents cadres théoriques sont historiquement situés. En s'intéressant à la formation et à la diffusion des concepts, l'approche généalogique cherche à souligner l'historicité des manières de penser et de problématiser de nouveaux objets de recherche. Ce faisant, elle suggère une vision des théories plus encastrées dans la société et tend à remettre en question l'idée d'une dichotomie claire entre théories et pratiques, qui prévaut souvent dans la littérature académique en matière de RSE (Acquier et Gond, 2006). L'intérêt d'une approche généalogique est double. Premièrement, elle offre une occasion de distanciation et de recul critique que ne permet pas une approche naturalisée ou ahistorique des concepts. Deuxièmement, en s'intéressant aux conditions d'émergence et à la diffusion des concepts de RSE, de performance sociétale et de stakeholders, l'approche vise aussi à s'interroger sur la pertinence et la transposabilité de cadres théoriques en vogue dans des

1. Cette approche a été proposée et théorisée par Michel Foucault. Pour une transposition de cette démarche dans le domaine de la gestion, le lecteur pourra se référer à l'ouvrage A. Hatchuel et al. (2005). 
contextes institutionnels et managériaux différents de ceux dans lesquels ils ont été historiquement formés.

Cette perspective généalogique se distingue ainsi d'autres histoires de la RSE comme celle de Carroll (1999) qui propose une histoire autonome des concepts théoriques, peu articulée de celle des pratiques d'entreprises. Elle est plus proche de l'histoire proposée par Pasquero (2005) qui a cherché à cerner l'identité du concept de RSE, de comprendre le contexte historique de sa naissance aux États-Unis et de sa diffusion dans d'autres contextes culturels. Elle s'en distingue cependant dans la mesure où nous essayons d'analyser plus systématiquement le raisonnement des chercheurs, en particulier la manière dont ces derniers ont problématisé leurs travaux par rapport à ceux de leurs contemporains et par rapport aux pratiques des entreprises et au contexte socioéconomique dont ils cherchaient à rendre compte.

Le plan de l'article suit un découpage chronologique en cinq parties. La première partie présente les premiers travaux qui ont visé à formaliser l'idée de responsabilité sociale aux États-Unis. La seconde partie analyse les premières formalisations de la RSE au cours des années 1950 et 1960. La troisième partie présente les travaux sur les capacités de réponse (responsiveness) apparus dans les années 1970. La quatrième partie présente les tentatives de synthèse théorique à travers les notions de performance sociétale de l'entreprise et les approches stakeholders. Enfin, la cinquième partie s'interroge sur les limites des approches stakeholders à l'aune des transformations récentes des pratiques en matière de RSE.

\section{L'ÉMERGENCE DE L'IDÉE DE « RESPONSABILITÉ SOCIALE » AUX ETATS-UNIS ${ }^{2}$}

Alors que le débat sur la responsabilité sociale de l'entreprise peut sembler relativement nouveau en Europe, la littérature économique et gestionnaire nord-américaine témoigne de l'existence et de la vigueur d'un tel débat aux États-Unis depuis le début du XIX $\mathrm{X}^{\mathrm{e}}$ siècle. À ce titre, l'analyse de travaux incluant une dimension historique (voir en particulier Bowen, 1953; Heald, 1961, 1970; Epstein, 2002) s'avère particulièrement riche en enseignements pour éclairer sous un angle nouveau les discussions actuelles sur la responsabilité sociale de l'entreprise.

Il apparaît que l'émergence de la grande entreprise va de pair avec une interrogation sur sa responsabilité. La notion de responsabilité sociale de l'entreprise, posant la question des rapports entre l'entreprise et la société, émerge à la fin du XIX $\mathrm{X}^{\mathrm{e}}$ siècle sous la forme mal définie d'un « mélange de bonnes intentions de la part d'hommes d'affaires [qui] produisit des résultats qui furent pour le moins confus " (Heald, 1961). Le débat se structure sous une forme plus formalisée au début du $\mathrm{XX}^{\mathrm{e}}$ siècle, à mesure que se généralise le modèle de la grande entreprise à actionnariat dispersé (Heald, 1970; Epstein, 2002) et la figure du dirigeant salarié non propriétaire.

2. Le lecteur intéressé par ces éléments, de même que le contenu de l'ouvrage fondateur d'Howard $\mathbf{R}$. Bowen (1953), (cf. partie II) pourra se référer pour plus de précisions à Acquier et Gond (2005). 
L'émergence de la grande entreprise à actionnariat dispersé a deux conséquences. Premièrement, elle diminue le contrôle que les actionnaires exercent sur les dirigeants. Deuxièmement, du fait de sa taille et de l'influence moins directe de ses propriétaires, l'entreprise change de nature pour devenir une institution à part entière. Elle interagit avec un ensemble de " groupes intéressés, incluant cette vague entité: la communauté sociale dans son ensemble " (Heald 1961).

La figure du dirigeant se transforme radicalement à mesure qu'aux dirigeants familiaux se substituent des juristes et ingénieurs formés dans les nouvelles Business Schools américaines ${ }^{3}$. Les programmes de formation, incluant des enseignements dans les domaines de l'économie et de la gestion, mais aussi des disciplines plus larges, développent une sensibilité des dirigeants aux relations entre leurs décisions et le fonctionnement plus général de la société. D'autre part, la nouvelle figure du dirigeant pose la question de l'identité, du rôle et de la déontologie du manager, que certains auteurs décrivent alors comme une profession (Malott, 1924; Donham, 1927; Calkins, 1946; Abrams, 1951).

À partir des années 1920, plusieurs dirigeants s'expriment publiquement sur leur responsabilité à l'égard de la société. Si « aucune doctrine clairement formulée de la responsabilité sociale n'avait émergé à la fin de la décennie ", les discours de l'époque sont très marqués par les concepts de « public service » et de " trusteeship » qui stipulent l'idée d'un contrat implicite, caractérisant la relation entre l'entreprise et la société (Heald, 1961, 1971). Ces débats sont certes controversés, mais sont portés par des grands dirigeants comme Henry Ford ou Alfred Sloan. De nouvelles pratiques d'entreprise se développent au cours de cette période, suivant une logique d'« essais-erreurs » (Heald, 1961). Des discours et pratiques relatifs à la responsabilité sociale se développent ainsi de manière précoce dans le milieu des affaires. Mais ce n'est que dans les années 1950 que des efforts significatifs de formalisation et de systématisation voient le jour à la frontière entre théologie, économie et gestion.

\section{1950-1965 : PREMIÈRES FORMALISATIONS DES RAPPORTS ENTRE ENTREPRISE ET SOCIÉTÉ}

\section{Les années 1950 : la formulation des termes du débat par des chercheurs}

Pour de nombreux auteurs, l'ouvrage théorique fondateur sur la question est celui de l'économiste Howard R. Bowen: Social Responsibilities of the Businessman (Bowen, 1953). Commandité par une organisation religieuse, le " département de l'église et de la vie économique ", ce livre fournit tout d'abord un témoignage historique très documenté, recense les discours des dirigeants sur la responsabilité sociale, entendue comme un ensemble d'obligations à l'égard de la société. Structuré autours des notions de stewardship, de trusteeship et de prise en compte des « groupes intéressés », qui résonnent étrangement avec certains concepts actuels (sta-

3. Aux États-Unis, la première business school fut fondée à l'université de Pennsylvanie en 1881. Deux autres furent créées en 1898 à Chicago et à l'université de Berkeley. En 1910, il y avait 14 écoles et en 1925 ce nombre se montait à 183 . 
keholders), on observe une importante interpénétration entre les discours analysés par Bowen et ceux de l'éthique religieuse. Mais l'ouvrage a, en fait, une portée plus importante que des considérations générales sur la responsabilité sociale. Il montre que les réflexions relatives à la responsabilité sociale s'inscrivent, durant les années 1950, dans le cadre de transformations assez profondes des modes de fonctionnement de l'économie américaine, et d'interrogations sur le modèle économique en place. Du fait de l'accroissement de la législation, de la concentration du pouvoir au sein de grandes entreprises mais aussi de l'émergence d'autres " groupes organisés " (syndicats, associations, etc.), l'économie américaine est mieux décrite sous la forme d'une « mixed economy » (p. 21), à mi-chemin entre le socialisme et le libéralisme. S'ils n'usent pas de leur pouvoir de manière responsable, les dirigeants risquent ainsi de voir s'accroître le contrôle social qui s'exerce sur eux, sous la forme de nouvelles régulations restreignant leur liberté d'action.

Pour conserver un système économique décentralisé et éviter de voir s'accroître les contraintes règlementaires restreignant leur liberté d'action, les hommes d'affaires doivent donc intégrer l'intérêt général à leurs décisions. Mais comment opérationnaliser ce nouveau modèle? Dans son ouvrage, Bowen discute de la « doctrine de la responsabilité sociale » (p. 6), alors en vogue dans les milieux d'affaires, et qui stipule qu'une approche basée sur l'engagement volontaire des hommes d'affaires pourrait suffire à résoudre des problèmes économiques et sociaux des États-Unis d'après guerre. Plutôt que d'accepter sans réserve cette proposition, Bowen s'interroge sur les conditions qui permettraient de faire de la responsabilité sociale un outil de régulation effectif de l'économie américaine. Il souligne, entre autres, la nécessité de développer de nouveaux outils de gestion, tels que l'audit social, ou la nécessité de réformes politique (développer de nouvelles instances de négociation entre les entreprises et leurs parties intéressées), de transformation des enseignements en gestion, etc. En trame de fond, Bowen développe ainsi une grille d'analyse de l'institutionnalisation de la responsabilité sociale (Acquier et Gond, 2005).

Un point intéressant de l'approche de Bowen est l'importance des considérations éthiques comme fondement de la responsabilité sociale des entreprises. Cet élément fondateur de la culture américaine, fondée sur l'appartenance à une communauté, souligne un point mis en évidence également par Weber dans son analyse des origines du capitalisme: la dynamique entrepreneuriale n'est pas réductible à un calcul économique; elle est mue par des fondements éthiques.

Dans une perspective plus centrée sur l'entreprise et ses dirigeants, Peter Drucker évoque les responsabilités des managers dans la conclusion de son ouvrage The Practice of Management, intitulée: The responsibilities of Management (Drucker, 1954). En une quinzaine de pages, il montre comment les enjeux sociaux et ceux de l'entreprise sont indissociables et en quoi les grandes entreprises, qui détiennent un pouvoir inégalé du fait de leur concentration inédite et de leur longévité, doivent assumer de nouvelles responsabilités. La démarche de Drucker consiste donc à fois à mettre en évidence la nécessité, pour les dirigeants, de tenir compte de l'environne- 
ment dans leurs pratiques, et de poser la question des principes qui doivent motiver les décisions de l'entreprise. Sur ce dernier point, Drucker, en ligne avec Clark (1916, 1939) ou Bowen (1953, 1955), rejette l'utilitarisme prôné par les économistes libéraux (Smith, Mandeville) comme principe de l'action managériale. Que cette proposition soit vraie ou fausse, aucune société ne peut être fondée sur l'idée selon laquelle l'agrégation des comportements égoïstes conduit mécaniquement à la maximisation du bien être public.

Ces deux approches sont intéressantes dans la mesure où elles portent en germe la nature du débat qui va se développer dans les années 1960 et comportent de nombreux points communs avec les recherches qui vont se développer ultérieurement, donnant naissance au champ Business and Society: l'idée qu'il existe un contrat implicite entre l'entreprise et la société, la question du pouvoir des grandes entreprises, le fondement normatif de la responsabilité de l'entreprise introduisant l'éthique comme une des variables du débat, la question de la déontologie des managers et de leurs principes d'action envers différents " groupes intéressés » (Abrams, 1951).

\section{Les premières formulations de la RSE}

Dans les années qui suivent, de nombreux auteurs formulent la responsabilité sociale de l'entreprise sur le registre de la contrainte morale ou réglementaire: reprenant l'idée d'un contrat entre l'entreprise et la société, la RSE est souvent définie comme un ensemble d'obligations incom- bant aux entreprises, qui s'additionnent à leurs obligations strictement financières et légales ${ }^{4}$.

La plupart des chercheurs partage une posture largement normative, et ne mobilisent pas les pratiques d'entreprise. Le débat se situe au niveau politique de la critique sociale de l'entreprise, et, dans leur contribution aux débats Business and Society, les auteurs adoptent le point de vue de la société plutôt que celui de l'entreprise. Ces points communs ne doivent cependant pas occulter le flou, l'hétérogénéité et la faiblesse des fondements théoriques de ces travaux. Pour reprendre l'analyse de Frederick, les premières approches de la RSE baignent dans un « brouillard normatif », donnant lieu à une éclosion de " débats philosophiques stériles " (Frederick, 1986). Par ailleurs, ces approches seront la cible de vives critiques de la part des défenseurs de la primauté absolue des actionnaires sur les autres membres de l'organisation. Si les tenants de la RSE ne contestent pas la mission de création de valeur de l'entreprise, accepter le principe d'une responsabilité sociale de l'entreprise revient à reconnaître l'existence d'autres acteurs et d'une mission audelà de la sphère économique, dont l'entreprise doit tenir compte dans le cours de son activité. C'est suffisant pour que Milton Friedman décrive la notion de Corporate Social Responsibility comme "profondément subversive ", considérant qu'" il existe peu de courants aussi dangereux pour les fondements même de notre société libre que l'acceptation, pour les dirigeants

4. Pour Davis, la RSE renvoie « aux décisions et aux actions prises pour des raisons au moins partiellement autres que les intérêts économiques ou techniques de l'entreprise " (Davis, 1960). Selon Carroll la RSE renvoie " aux attentes de la société envers l'entreprise sur le plan économique, légal, éthique et discrétionnaire " (Carroll, 1979). 
d'entreprise, d'une responsabilité autre que celle de maximiser le rendement de l'argent de leurs actionnaires "(Friedman, 1962).

\section{1965-1980 : UN MOUVEMENT DE RATIONALISATION DES RELATIONS ENTRE ENTREPRISE ET SOCIÉTÉ}

\section{Début 1960 - milieu 1970 : la légitimité de l'entreprise en question}

Si l'ensemble des institutions américaines (entreprises mais aussi l'État) avait traversé, à partir de la fin de la Seconde Guerre mondiale, une période d'amélioration continue de leur image, le milieu des années 1960 marque un retournement brutal et durable. À l'occasion de la réédition d'une note de recherche, William Frederick revient sur ce contexte en soulignant la conflictualité des relations entre les entreprises et le reste du corps social :

" Mon intuition est qu'il est très difficile, aujourd'hui, pour une personne n'ayant pas vécu le tumulte social des années 1960 et du début des années 1970 , de saisir l'effet de tremblement de terre suscité par ces événements sur le milieu des affaires et la prise de conscience de ces enjeux dans les entreprises. L'entreprise se retrouvait violemment projetée dans un maelström social, dans lequel beaucoup remettaient en cause non seulement sa légitimité, mais aussi fondamentalement son droit à l'existence. " (traduction) (Frederick, 1994).
Ainsi, les entreprises se trouvent confrontées à de nouveaux mouvements contestataires, qui vont donneront lieu à une importante vague de régulation au cours des années 1960 et dans la première moitié des années $1970^{5}$ : en publiant Unsafe at Any Speed en 1965, Ralph Nader met violemment en cause les pratiques des constructeurs automobiles (en particulier General Motors) et leur acharnement à éviter l'introduction de normes de sécurité dans leurs produits (Nader, 1965). La parution en 1967 du Nouvel État Industriel de Galbraith, et la notion de filière inversée, témoigne aussi de la stigmatisation d'un pouvoir excessif de la grande entreprise vis-à-vis de son environnement et de ses clients (Galbraith, 1969). Au cours des années 1970 est créée une agence publique de protection des consommateurs, la Consumer Product Commission (CSPC). Au-delà de la montée des mouvements consuméristes, les années 1960 sont marquées par l'émergence de préoccupations relatives à l'égalité des droits et aux discriminations (Vogel, 1986). D'un point de vue institutionnel, la période est marquée par la création d'Amnesty International en 1961 et la mise en place d'une commission américaine sur l'égalité des opportunités d'emplois (US Equal Employment Opportunity Commission) en 1964. De plus, la question de la sécurité au travail fait aussi l'objet de réglementation, avec la création dans les années 1970 de l'Occupational Safety and Health Administration. Enfin, on observe une attention nouvelle aux enjeux environnementaux. La publication

\footnotetext{
5. Ces critiques et ces mouvements ne sont pas spécifiques aux États-Unis mais ils suscitent là-bas un débat à la mesure de la place qu'occupe, à la différence de la France par exemple, l'entreprise dans la société américaine.
} 
de l'ouvrage Silent Spring par R. Carson (1962), qui met en cause les dégâts irrémédiables causés par l'usage des pesticides, fait voler en éclat l'idée selon laquelle l'environnement a une capacité d'absorption infinie (Carson, 1962). De nouvelles ONG sont créées, comme le WWF en 1961 ou Greenpeace en 1975. Ces préoccupations environnementales se traduisent sur le plan institutionnel par l'adoption du Clean Air Act en Californie en 1963, du Wilderness Act en 1964 et la création de l'Agence pour la protection de l'environnement (EPA) au début des années 1970.

L'entreprise devient ainsi l'objet de violentes controverses et la cible de démarches activistes, dont les pressions peuvent résulter dans de nouvelles réglementations encadrant l'action des entreprises.

\section{De la responsabilité à la réponse (responsiveness) : le développement d'une approche managériale de la RSE}

Devant l'instabilité et la complexité nouvelle de l'environnement, la question de la RSE ne renvoie plus simplement à une question d'éthique et de choix du dirigeant. Il s'agit de repérer, pour les entreprises, d'où viennent ces forces et de comprendre comment l'entreprise peut y apporter un traitement systématique. Cette question managériale se traduit par la création de nouvelles figures d'acteurs (le « social issue specialist $»$ ), de nouveaux outils et de nouvelles règles au sein des entreprises, notamment concernant les pratiques d'audit social (Bauer et Fenn, 1972; Dierkes et Bauer, 1974). Ainsi, Ackerman et Bauer (1976) rapportent qu'en 1973, " une majorité des très grandes entreprises avaient "audité" leur performance sociale au cours des douze derniers mois " (p. viii), alors même que ces démarches étaient inexistantes quelques années auparavant.

Le courant « Corporate Social Responsiveness " naît dans ce contexte. II trouve son socle dans un projet de recherche développé au sein de la Harvard Business School en 1971, sous l'autorité de Raymond A. Bauer (Ackerman, 1975). Fídèle à la tradition de la Harvard Business School, ce programme se développe, entre 1972 et 1974 , autour de différentes études de cas et de nombreuses interviews de cadres dirigeants dans plus de 40 grandes entreprises. S'éloignant volontairement de débats généraux sur le besoin de refonder une théorie de l'entreprise, les auteurs prônent une démarche pragmatique. Deux ouvrages de synthèse sont publiés à un an d'intervalle: The Social Challenge to Business (Ackerman, 1975) et Corporate Social Responsiveness (Ackerman et Bauer, 1976).

Ces travaux, qui restent selon nous d'une richesse inégalée, sont les premiers à analyser les processus de déploiement de telles démarches et à proposer une analyse détaillée des politiques et instruments mis en cuvre par les entreprises en matière de RSE. Par ailleurs, ils font partie des rares approches à resituer la complexité de l'action du dirigeant, à ne pas traiter l'organisation comme une boîte noire mais à s'intéresser à sa structure, ses outils, et à intégrer des éléments de théories des organisations pour la compréhension de ses dynamiques. Dans leur analyse, Ackerman et Bauer (1976) replacent l'émergence des débats sur la responsabilité sociale de l'entreprise dans le cadre plus global d'une crise d'ensemble des institutions américaines et d'un " agenda for a humanized society ». Audelà de la capacité des entreprises à prendre 
en charge de nouvelles questions sociales, le terme de corporate social responsiveness renvoie à la capacité des firmes multidivisionnelles à piloter le changement et à renouveler leurs modes d'action de manière régulière: «Alors que notre attention s'est portée sur les enjeux sociétaux (social issues), nous pensons que le phénomène général que nous étudions est la capacité d'apprentissage de la grande entreprise moderne à institutionnaliser la nouveauté (learning to institutionalize novelty). [...] Nous sommes les témoins de l'apparition d'entreprises réactives et sensibles à leur environnement, qui devraient, si le processus est mené à bien, être de plus en plus aptes à prendre en charge une grande diversité d'enjeux, qu'ils soient économiques ou sociétaux. " (traduction) (Ackerman et Bauer, 1976, p. 13).

\section{Le développement d'une approche stratégique des questions sociales}

Pour Ackerman et Bauer, les questions sociales ne sont pas fondamentalement différentes des autres questions traditionnelles de management. Par analogie à la notion de cycle de vie des produits en marketing, les auteurs proposent un modèle générique de développement des enjeux, le cycle de vie des « social issues », constitué de trois phases successives. Au cours de la première étape, l'enjeu reste de l'ordre du signal faible. Les questions centrales ont trait à la formulation de la question, à sa pertinence, sa compréhension et la prise de conscience du public. Dans une seconde étape, le problème reste émergent et non résolu, mais les incertitudes se déplacent: quels sont les acteurs concernés, comment résoudre le problème, quelles sont les compétences nouvelles à développer et comment modi- fier le comportement des acteurs (par l'incitation ou la contrainte règlementaire) ? Dans une troisième phase, la situation est maîtrisée: le problème est caractérisé et les solutions identifiées, et l'impact économique (coût des mises aux normes, etc.) est mesurable pour l'entreprise. Par le biais de normes ou de nouvelles lois, des standards de comportement ont été établis, reflétant des attentes explicites des entreprises en matière de RSE. Suivant les auteurs, l'entreprise peut adopter une position de pionnier ( « lead ») ou de suiveur ( lag »); cette position ayant pour effet d'anticiper ou de retarder l'application de solutions socialement responsables dans l'entreprise.

\section{La capacité à orchestrer un processus de changement organisationnel}

Cette phase de diagnostic et de positionnement stratégique n'est qu'un des éléments dans le processus de développement de capacités de réponse de l'entreprise. Ainsi les auteurs soulignent la nécessité d'un processus de changement organisationnel plus large, qu'ils découpent en trois étapes (Ackerman, 1973).

La première étape correspond à une prise de conscience de la part du dirigeant qui formule une politique d'entreprise (policy statements) sur la question. Dans cette phase d'engagement du dirigeant, la question est traitée comme un problème politique (policy problem).

La seconde phase (learning) est centrée sur un effort de compréhension et de caractérisation du problème, et sur l'identification d'une réponse adaptée de la part de l'entreprise. D'une approche politique, le problème devient technique. Cette étape est souvent marquée par la création d'un poste de spécialiste, hors de la ligne hiérarchique, 
qui apporte à l'entreprise un bagage technique qui lui fait défaut. Dans une position d'intermédiaire entre la direction, l'environnement de l'entreprise et les middle managers des différentes divisions, le " special issue specialist » doit être à même de développer des capacités de négociation et de politique pour pouvoir s'ancrer dans l'entreprise et générer de nouvelles pratiques.

Dans une troisième phase (organizational involvement and commitment), l'enjeu porte sur la généralisation de la démarche, son appropriation par les acteurs opérationnels, et l'institutionnalisation d'une démarche de responsiveness au sein de l'entreprise.

Au-delà de cette vision en trois étapes dont les aspects mécaniste et simplificateur sont discutables, ces travaux ont le mérite de développer une théorie de l'organisation multidivisionnelle (qu'il serait trop long de détailler ici), et de proposer un cadre d'analyse systématique de l'intégration de la responsabilité sociale dans l'organisation de l'entreprise.

\section{La portée du courant Responsiveness}

Dans son ensemble, la vague de la Corporate Social Responsiveness a eu un impact contrasté sur le champ de recherche de la RSE. L'orientation managériale et l'analyse en termes de processus a influencé les travaux suivants de deux manières. Premièrement, elle a conduit à la requalification par certains auteurs (Wartick et Cochran, 1985; Vogel, 1986; Wartick et Rude, 1986; Wood, 1991b) du champ «Entreprise et Société » en «Questions Sociales dans l'Entreprise » (Social Issues in Management). Plus clairement intégré aux cadres stratégiques et organisationnels les plus courants, ce label constitue toujours l'une des divisions de l'Academy of Management. Deuxièmement, elle a une filiation avec les premières approches stakeholders à dominante managériale et sera réappropriée au sein du concept plus large et englobant de performance sociétale de l'entreprise. Pourtant, ce courant de recherche restera d'une portée limitée et ne survivra pas à la décennie des années 1980, marquées par le recul de l'engagement des grandes entreprises américaines en matière de RSE et la disparition des jeunes directions des affaires sociales.

\section{1980-2000 : TENTATIVES DE SYNTHÈSE THÉORIQUE. LA QUETE DE CONCEPTS INTÉGRATEURS}

La période s'étalant des années 1980 à la fin des années 1990 est marquée par le recul des pratiques d'entreprises en matière de RSE. Dans un contexte socio-économique moins favorable, les chercheurs du champ Business and Society cherchent à maintenir leur position. Cette période est ainsi marquée par une volonté claire de consolidation et d'unification d'approches qui semblaient de plus en plus hétérogènes. Les concepts de performance sociétale de l'entreprise et de stakeholder vont donc se développer dans une quête de théorie intégratrice des précédents travaux.

\section{La performance sociétale de l'entreprise (Corporate Social Performance)}

En consultant les articles les plus significatifs sur la notion de Corporate Social Performance (Carroll, 1979; Wartick et Cochran, 1985; Carroll, 1991; Wood, 1991a; Swanson, 1995; Carroll, 1999; 
Swanson, 1999), il est étonnant de constater que ces travaux ne sont pas consacrés à interroger la notion de " performance ", à sa transposition au champ de la responsabilité sociale ou à la définition de nouveaux critères de performance intégrant des dimensions sociales et environnementales. La contribution de ces auteurs est d'une autre nature, et se comprend plutôt comme un effort de fédération et de mise en cohérence d'approches hétérogènes. Ainsi, Carroll (1979) définit la performance sociétale de l'entreprise comme renvoyant à un ensemble de trois dimensions: les principes de responsabilité sociale adoptés par l'entreprise (il renvoie ainsi aux premiers débats sur la responsabilité sociale de l'entreprise), les modes de réponses et processus de déploiement (on retrouve ici la deuxième vague de recherches autour de la notion de responsiveness), et l'ensemble des " enjeux sociaux " (social issues) auxquels l'entreprise est confrontée. La logique des autres travaux est relativement proche, présentant la performance sociale de l'entreprise comme un ensemble de principes, de processus et de résultats (Wood, 1991a; Swanson, 1995), dans une volonté similaire de synthèse et d'intégration théorique.

\section{Logique et limites des études sur la relation entre performance sociale et financière de l'entreprise}

Les premières recherches posant la question des performances financières des entreprises dites " socialement responsables " émergent au début des années 1970 ((Moskotitz, 1972). De nombreuses études quantitatives ont été menées depuis lors dans le but d'établir un lien statistique (et en trame de fonds des lois générales) entre la responsabilité sociale et la performance financière de l'entreprise. S'il n'est pas utile d'en effectuer ici une revue détaillée (le lecteur intéressé pourra se référer à différents travaux de synthèse (Margolis et Walsh, 2003; Orlitzky et al., 2003; Allouche et Laroche, 2005; Gond, 2006)), on peut retenir que : 1) ce courant de recherche est toujours vivace et continue d'alimenter de nombreux travaux, 2) ces travaux ne sont pas indépendants de la volonté de légitimer les pratiques de RSE à l'heure de la création de valeur actionnariale et 3 ) les différents efforts de raffinement méthodologique et statistique ne sont pas parvenus à établir de lien évident et à clore les controverses autour du lien entre performance financière et performance sociale de l'entreprise.

\section{Les approches stakeholders}

Le concept œcuménique de stakeholder constitue une deuxième occasion de synthèse théorique majeure. Ce concept est doublement intéressant. Premièrement, il bénéficie d'une large diffusion au sein des milieux académiques, comme en témoignent des publications mobilisant la théorie des parties prenantes dans les revues académiques internationales les plus sélectives en management stratégique 6 . De plus, le concept de stakeholder apparaît aujourd'hui comme le concept fédérateur pour mener à bien une unification voire une refondation théorique du champ Business and Society (Freeman, 1994, 1999; Andriof et al., 2002; Andriof et al., 2003). Deuxième-

6. Academy of Management Review, Strategic Management Journal, Journal of Management Studies, etc. 
ment, le concept de stakeholder se distingue par son appropriation massive et inédite dans le champ des pratiques managériales. Ainsi, le management des parties prenantes occupe une place centrale dans la conceptualisation et la mise en ouvre de la RSE au sein des entreprises. Au-delà des entreprises, le concept de stakeholder semble aussi se diffuser dans l'ensemble de la société à travers la montée en puissance des «groupes concernés " mais aussi l'émergence d'une démocratie participative (Callon et al., 2001).

C'est dans le sillon des approches managériales de la Corporate Social Responsiveness que le terme de stakeholder va se développer, à partir de la fin des années 1970 et du début des années 1980 (Charan et Freeman, 1979; Sturdivant, 1979; Management Review, 1980). Ces premiers travaux font écho à un " projet stakeholder ", mis en place en 1977 à la Wharton School, au sein du Centre de recherche appliqué (Applied Research Center). "L'objectif de ce projet était de réunir de nombreux courants de pensée ${ }^{7}$ et de développer une théorie du management qui permette aux cadres dirigeants de formuler et de mettre en place des stratégies d'entreprises dans des environnements turbulents. " (Freeman et Reed, 1983). L'originalité de ces approches est d'inclure au cœur de l'analyse les « groupes adverses ». Dans ce cadre, l'environnement est perçu comme menaçant pour l'entreprise, et la notion de stakeholder est définie de manière défensive. À ce titre, Sturdivant emprunte à Emshoff et Freeman une définition du stakeholder comme « n'importe quel groupe dont le comportement collectif peut affecter de manière directe le futur de l'organisation, mais qui n'est pas sous son contrôle direct " (Emshoff et Freeman, 1978) ${ }^{8}$. L'entreprise devenant un objet de lobbying (Vogel, 1978), les problématisations initiales de la notion de stakeholder sont largement marquées par les possibilités d'influence de ces acteurs et les relations de dépendance de l'entreprise vis-à-vis de ces derniers. Deux autres éléments témoignent de l'influence des approches responsiveness sur les premiers travaux de ce courant de recherche. Premièrement, les démarches stakeholders initiales délaissent la recherche de principes normatifs de responsabilité de l'entreprise et adoptent une démarche pragmatique, en justifiant les pratiques de stakeholder management sur la base de leur efficacité. Deuxièmement, pour ces auteurs, la gestion des stakeholders procède par adaptation et extension des cadres et outils traditionnels de management (Freeman et Reed, 1983; Freeman, 1984).

\section{Les premiers développements conceptuels}

L'ouvrage Stakeholder management, a strategic approach de E. Freeman va marquer une première étape de construction conceptuelle en ancrant la gestion des parties prenantes à un niveau stratégique (Freeman, 1984). Freeman propose le concept de stakeholder comme mode de

\footnotetext{
7. Selon Freeman (1984) les démarches de stakeholder management trouvent notamment leurs racines dans les travaux de I. Ansoff et de R. Stewart au département planification de Lockheed et à l'ouvrage Corporate Strategy de Ansoff (1965).

8. Freeman (1984) accorde la paternité de la notion de stakeholder à une note interne du Stanford Research Institute (SRI, organisme de recherche et de conseil) de 1963.
} 
Figure 1 - Une représentation des modèles d'intention

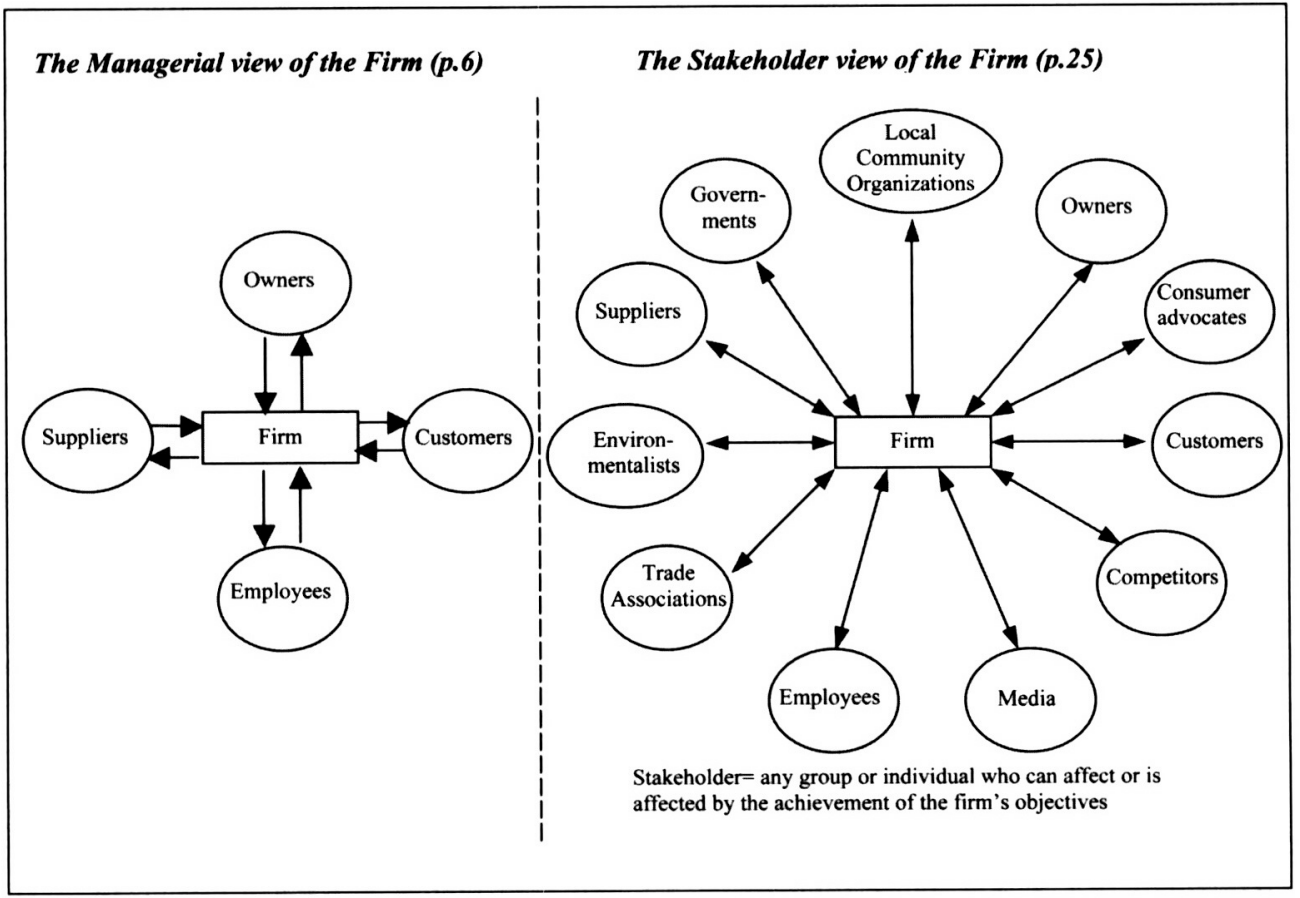

Source: à partir de Freeman (1984).

représentation de l'organisation et de relecture de sa stratégie (l'entreprise est au centre d'une roue, reliée à ses stakeholders par des rayons). Les stakeholders sont définis comme « tout groupe ou individu qui peut influencer ou être affecté par l'atteinte des objectifs de l'organisation " (cf. figure 1). S'il cherche à réunir différentes approches de la stratégie dans son ouvrage Stakeholder Management, a Strategic Approach, les deux branches influençant le plus fortement l'ouvrage sont la planification stratégique (Ansoff, 1965) et la théorie de la dépendance en ressources (Pfeffer et Salancik, 1978).

Suivant une approche managériale, il est important de souligner que Freeman déve- loppe une approche utilitariste et instrumentale de la gestion des stakeholders (Walsh, 2005) : ainsi, la prise en compte des demandes des stakeholders n'est pertinente que si elle est associée aux objectifs de la firme. La gestion des stakeholders n'est qu'un moyen de préserver ou de redonner des marges de manœuvre à la firme et ses managers. De même, la question de la remise en cause des structures de gouvernance actionnariale et de leur démocratisation n'est évoquée que dans le dernier paragraphe de l'ouvrage, sous la rubrique Future Research, déconnectée du reste de l'argumentation. Ainsi, selon Andriof et al. (2002b) « la pensée des stakeholders s'est déployée dans une direction que beaucoup 
considèrent comme un chemin différent du courant Business and Society " (traduction, p. 13), et l'intégration de considérations éthiques se fera " par ajout » (« ethics being added $»$, p. 13) dans un deuxième temps, au cours des années 1990 (Andriof et al., 2002).

Comme le rappellent Martinet et Reynaud (2004), l'apport de l'approche ouverte par Freeman est de proposer un cadre intégrateur au management stratégique dépassant les conceptions restrictives de la stratégie focalisées sur les rapport entre les managers et les actionnaires (la théorie de l'agence) ou les rapports entre l'entreprise et les acteurs-clés de l'environnement (les stratégies concurrentielles à la Porter). De manière significative, en parallèle des travaux de Freeman sur les stakeholders se développe en France, à partir de la fin des années 1970, une série de travaux qui cherchent à refonder le champ du management stratégique en intégrant des variables socio-politiques dans l'analyse (Martinet, 1984 ; Jarniou, 1981 ; Pasquero, 1980). À côté des forces concurrentielles, l'entreprise fait face à une multitude de pressions socio-politiques, c'est-à-dire des exigences formulées par une partie de la société environnante envers l'entreprise afin que cette dernière intériorise des coûts sociaux nouveaux, imputables ou non à ses activités de production (Pasquero, 1980). Dans cette perspective, l'entreprise est simultanément un agent de production, une orga- nisation sociale et un système politique (Martinet, 1984). L'ambition théorique proposée par ce courant de recherche n'est pas de développer une stratégie sociétale autonome mais bien d'intégrer la responsabilité sociale dans un cadre conceptuel et méthodologique étendu de la stratégie permettant de mieux prendre en compte la question de son acceptabilité sociale (Martinet, 1984).

\section{La diversité des approches stakeholders}

Cependant, ces premiers travaux ne constituent pas un corpus théorique abouti et vont donner lieu à des développements hétérogènes. Ainsi, " le travail de Freeman, même s'il reconnaît formellement l'importance de composantes de l'entreprise autres que les actionnaires, laisse flou le statut du concept de stakeholder en tant que théorie " (Jones, 1995). De fait, la notion de stakeholder va donner lieu à un foisonnement de travaux et d'approches hétérogènes : « quiconque analyse d'un œil critique cette littérature large et évolutive observera que les concepts de stakeholder, de modèle stakeholder, de stakeholder management et de stakeholder theory sont expliqués et utilisés par des auteurs variés de manière très différente, et sont soutenus (ou critiqués) sur la base de justifications et d'arguments divers et souvent contradictoires " (Donaldson et Preston, 1995) (voir encadré ci-après). 


\section{LES APPROCHES STAKEHOLDERS, QUELLE COHÉRENCE? QUELLE UNITÉ?}

Dans leur article The stakeholder theory of the corporation: concepts, evidence and implications, Donaldson et Preston proposent de distinguer les branches descriptives, instrumentales et normatives de la théorie des stakeholders:

- les approches descriptives (ou analytiques) visent à comprendre dans quelle mesure les managers répondent à différents stakeholders et agissent en fonction de leur intérêt. Il s'agit alors de modéliser la firme dans son interaction avec ses différents stakeholders. On peut ranger dans cette catégorie les travaux visant à comprendre comment les différents attributs des stakeholders peuvent influencer leur prise en compte par les dirigeants (cf. notamment Mitchell et al., 1999; Mitchell et al., 1997) qui retiennent comme variables l'urgence de la demande, le pouvoir et la légitimité des stakeholders), où les travaux montrant comment la configuration du réseau de stakeholders et l'importance relative de chaque groupe évolue en fonction du cycle de vie de l'organisation (Jawahar et Mclaughlin, 2001), les travaux de Frooman (1999) sur les stratégies d'influence des stakeholders, l'analyse du réseau entreprise/stakeholders et son influence sur les stratégies de réponse (Rowley, 1997), la compréhension des processus de mobilisation des stakeholders (Rowley et Moldoveanu, 2003), etc. - la branche instrumentale étudie les conséquences organisationnelles (souvent en termes de performance financière, de croissance ou de stabilité) résultant de la mise en place de pratiques de gestion des stakeholders dans l'entreprise (Clarkson, 1995; Waddock et al., 1997a ; Waddock et Graves, 1997b) ;

- enfin, les approches normatives visent à « identifier les principes moraux et philosophiques orientant l'activité et la direction des entreprises " (c'est-à-dire la prise en compte des demandes et de l'intérêt intrinsèque de l'ensemble des stakeholders légitimes) et à proposer une justification du cœur normatif de la théorie. Pour Donaldson et Preston, la théorie moderne des droits de propriété peut servir de justification des principes moraux de la stakeholder theory ${ }^{9}$.

Cette typologie, largement reprise dans la littérature, a aussi été l'objets de débats multiples. Les efforts d'unification (Jones et Wicks, 1999) ne sont pas acceptés par l'ensemble des auteurs et sont marqués par des contradictions internes (Donaldson, 1999; Gioia, 1999; Trevino et Weaver, 1999). Par exemple, Mitchell et al. (1997), dans un article lui aussi très cité, avancent que la prise en compte des stakeholders par l'entreprise dépend des variables de pouvoir, de légitimité et d'urgence. Les parties prenantes ont d'autant plus de chance de recueillir une attention de la part des dirigeants qu'elles cumulent ces trois attributs. Cela signifie qu'un stakeholder légitime mais sans pouvoir et dont la demande n'est pas urgente aura peu de chance d'être pris en compte. Ainsi, les résultats des approches descriptives met-

9. Pour une revue des justifications normatives de la stakeholder theory, on pourra se référer aux travaux de Phillips (2003; Phillips et al., 2003) ainsi qu'à l'ouvrage collectif coordonné par Bonnafous-Boucher et Pesqueux (2006). 
tent-elles en évidence des contradictions entre les pratiques réelles et le cœur normatif de la théorie (Trevino et al., 1999). Du fait de ces contradictions internes, il est difficile de considérer que cet ensemble de courants forme une théorie unifiée des parties prenantes. Le management des stakeholders est mieux décrit comme une « tradition de recherche, incorporant des théories multiples et variées qui se concentrent sur le même domaine de phénomènes observé ou postulé, ou à des ensembles reliés de questions ou de problèmes " (Trevino et al., 1999, p. 224).

Alors que Donaldson et Preston proposent que la branche normative soit à la fois distincte et en surplomb des autres approches, Freeman considère que cette logique tend à alimenter la « thèse de la séparation », c'est à dire l'idée selon laquelle on pourrait dissocier les dimensions managériales et morales de toute action gestionnaire, que l'on peut considérer de manière autonome les sphères économiques et éthiques de la vie des affaires. Il souligne au contraire que toute théorie du management comporte une dimension morale, même si celle-ci reste bien souvent implicite (Freeman, 1994). Dans ce cadre, " la stakeholder theory se distingue [des approches traditionnelles en stratégie] en ce qu'elle aborde explicitement les questions morales et les valeurs comme un aspect central des organisations » (Phillips et al., 2003). Abandonnant la thèse de la séparation, les approches stakeholder deviennent alors un « genre narratif » (Freeman, 1999), une manière de réintégrer dans l'analyse les dimensions morales et managériales de l'action gestionnaire. Dans ce cadre, il n'y a plus une mais plusieurs « théories " stakeholders possibles, avec des cours normatifs divergents. Ainsi, on pourrait considérer les approches actionnariales de la firme comme une approche stakeholder, à condition de réintégrer de manière explicite leurs fonde- ments moraux et de discuter leurs conséquences. Selon Freeman, une telle rẻintégration des aspects normatifs et descriptifs donnerait une dimension plus pragmatique et opérable aux démarches éthiques, jusqu'alors centrées sur la recherche d'un socle éthique autonome et déconnecté des pratiques réelles des entreprises.

\section{Quatre propositions qui réunissent l'ensemble des approches stakeholders}

Au-delà des divergences que l'on peut repérer entre les différentes approches stakeholders, il nous semble possible d'identifier quatre propositions minimales qui font consensus au sein de ce courant de recherche (à partir de Aggeri et Acquier, 2005):

\section{Pl : l'entreprise a des stakeholders qui ont des requêtes à son égard}

Cette proposition est le point de départ de tous les travaux sur les stakeholders. Elle exprime la représentation relationnelle et contractuelle de la firme sur laquelle se fondent les travaux. Dans une perspective de système ouvert, l'entreprise entre en relation (explicite, implicite, volontaire ou pas) et en négociation, dans le cours de ses activités, avec différents stakeholders, qui ont une identité et des préférences identifiables, et dont la participation est nécessaire au bon fonctionnement de l'entreprise. 
P2: tous les stakeholders n'ont pas la même capacité d'influence sur l'entreprise Même si les critères de hiérarchisation ne font l'objet d'aucun consensus, la plupart des auteurs acceptent l'idée que tous les stakeholders n'ont pas la même importance pour l'entreprise et que celle-ci, ne pouvant répondre à toutes les exigences formulées à son égard, doit sélectionner celles auxquelles elle doit répondre.

\section{P3: la prospérité de l'entreprise dépend} de sa capacité à répondre aux demandes de stakeholders influents et légitimes

Cette proposition renvoie bien à l'importance des concepts de légitimité et de pouvoir dans l'analyse des relations entre l'entreprise et ses parties prenantes. On retrouve ici l'idée selon laquelle la responsabilité de l'entreprise s'exerce de manière contingente en fonction de chaque demande et de chaque stakeholder.

\section{P4: la fonction principale du management} est de tenir compte et d'arbitrer entre les demandes potentiellement contradictoires des stakeholders

Dans la perspective relationnelle précédemment évoquée, manager les stakeholders consiste à identifier les stakeholders légitimes et influents, à cartographier les pressions exercées par ces derniers, puis à déployer des démarches managériales aux différents niveaux de l'organisation.

\section{1995-2005 : TRANSFORMATION DES PRATIQUES DE RSE ET LIMITES EXPLICATIVES DES APPROCHES STAKEHOLDERS}

Il est significatif de remarquer que les approches stakeholders, dont les principaux développements se sont faits en relative déconnexion avec les pratiques d'entreprise, sont aujourd'hui largement diffusées, non seulement dans les discours d'entreprise, mais également dans des outils de management des stakeholders et d'engagement entre l'entreprise et ses parties prenantes (stakeholder panels, partenariats, etc.). La tentation est alors de considérer que le cadre d'analyse des stakeholders constitue le cadre d'analyse gestionnaire de référence des pratiques d'entreprise en matière de développement durable ou de RSE. Dans cette dernière partie, nous allons montrer que cette réappropriation des concepts théoriques par les praticiens s'explique par d'autres raisons que la pertinence de la théorie. Nous essayerons de montrer que les approches théoriques présentent un certain nombre de limites et d'angles morts, invitant à élargir ou renouveler les questions de recherche et cadres d'analyse de la RSE.

\section{La transposition des concepts théoriques dans la sphère managériale : des mécanismes de diffusion inexpliqués}

Lorsque, dans les années récentes, les spécialistes de la théorie des stakeholders commencent à relater et à formaliser ces nouvelles pratiques, ils considèrent que la notion d'engagement marque une nouvelle étape de la théorie des parties prenantes (c'est le ccur de l'ouvrage collectif Unfolding Stakeholder Thinking: Theory, Responsibility and Engagement, 2002). Constatant l'émergence de processus de collaboration et d'engagement entre les entreprises et leurs stakeholders, les auteurs soulignent un déplacement de logique et l'émergence d'un nouveau paradigme, passant de la gestion des stakeholders (où l'en- 
treprise située au centre d'un réseau de parties prenantes, qu'elle gère de manière défensive) à la gestion des relations de l'entreprise avec ses stakeholders, dans un système polycentré de relations complexes (Rowley, 1997), suivant une approche moins réactive (Andriof et al., 2002).

Si ces propositions de réorientation théorique paraissent de bon sens, il est surprenant de voir que les auteurs n'interrogent pas l'origine de ces pratiques et combien ont-ils tendent à reproduire, avec peu de réflexivité, les discours dominants des consultants sur le développement durable. Ainsi, ils véhiculent, sans jamais l'interroger, l'idée d'un «business case» de la gestion des stakeholders, c'est-à-dire d'un argumentaire économique du bien-fondé de la RSE pour les entreprises, à travers des affirmations telles que: "si, dans le passé, l'attention s'est focalisée sur l'accroissement de la valeur pour l'actionnaire, elle porte désormais sur l'engagement avec les stakeholders en vue d'une création de valeur à long terme." (traduction) (Andriof et al., 2002, p.9). Or quelle est la validité d'un tel énoncé?

À aucun moment, les auteurs ne font état, dans l'èmergence de ces nouvelles formes de relations entre entreprises et parties prenantes, de l'influence des consultants, notamment britanniques, qui semble pourtant essentielle (voir Aggeri et al., 2005, partie 1, chap. 2). Ces consultants ont joué un rôle-clé de passeur entre le monde académique et celui des entreprises, en transponsant un certain nombre de concepts théoriques et en les reformulant dans un langage accessible aux managers (voir Elkington, 1998 ; Zadek, 2004). Ils ont également contribué à l'identification, à la formalisation et à la diffusion des «bonnes pratiques " des entreprises pionnières. Ils ont ainsi participé à l'invention d'un nouveau langage managérial qui se caractérise par l'hybridation du concept de RSE avec celui de développement durable, libérant la RSE de sa dimension militante, trop explicitement éthique qui sied mal aux cultures européennes.

On trouve les traces de ces discours dans les ouvrages de vulgarisation en matière de développement durable ou de RSE (WBCSD, 2002 ; Preston et al., 2002). Sans en faire une analyse détaillée, une des propriétés intéressantes de ce discours est qu'il fonctionne selon une logique de "pansement symbolique " (Laufer, 1996), car il s'agit de réparer et de préserver le système tout en intégrant les critiques sociales concernant les crises du capitalisme et leurs modes de résolution. Ainsi ce discours dramatise les crises et les dysfonctionnements du système capitaliste (crise de la gouvernance et du contrôle des dirigeants, cynisme des salariés, problèmes environnementaux et sociaux globaux) pour justifier l'urgence d'un changement de paradigme s'appuyant sur de nouveaux concepts (comme la « triple bottom line " (Elkington, 1998; Norman et al., 2004) ou le nouveau concept en vogue de corporate citizenship (Scholte, 2001; Matten et Crane, 2005), mais sans remettre fondamentalement en question les institutions existantes qu'elles permettent de préserver. Une des promesses de ces discours est une sorte de capitalisme réconcilié avec l'ensemble de la société et de ses parties prenantes. En effet, à en croire ces discours, la RSE bénéficierait à l'ensemble des parties prenantes: pour l'actionnaire, les pratiques de reporting environnementales et sociales doivent déboucher sur une transparence accrue, permettre de mieux valoriser les 
risques ou de mieux appréhender la valeur du « goodwill » de l'entreprise. De même, l'engagement en faveur du développement durable est sensé bénéficier à l'entreprise via son image institutionnelle, le moral de ses salariés, leur rapport au travail et à l'entreprise. Par ailleurs, ce type d'engagement serait susceptible de diminuer les risques de crise institutionnelle et les mouvements de boycotts de consommateurs. Enfin, pour l'État, les ONG et la société civile, l'engagement des entreprises est une promesse de relations apaisées et plus coopératives avec l'entreprise, et un pas vers la résolution constructive, partenariale, innovante et synergique des problèmes sociaux et environnementaux.

La main de ces consultants est invisible dans une théorie naturalisée des stakeholders où le développement de nouvelles pratiques de gestion des parties prenantes est présenté comme une condition de survie des entreprises modernes, découlant rationnellement de l'analyse des pressions externes qui s'exercent sur les entreprises. Il y a un risque, nous semble-t-il, à ne considérer que la " sphère des pratiques rationalisées "), c'est-à-dire les pratiques observables, mais sans tenir compte du processus de rationalisation, de la manière dont les doctrines de management des stakeholders sont fondées et les démarches de normalisation qui les sous-tendent. Ce risque est, pour les chercheurs, de se retrouver prisonniers des représentations des praticiens qui inventent ces démarches et participent aux régulations qu'ils contribuent à mettre en place.

\section{La question des modes de régulation}

Une seconde critique des approches stakeholders concerne leur caractère firmo-centré. En effet, celles-ci posent la question de la capacité de la firme à gérer ses relations avec les acteurs de son environnement. Comme la plupart des théories du management stratégique, elles restent majoritairement centrées sur le noyau stratégique de l'entreprise. La portée de cette unité d'analyse est limitée pour l'analyse de certains enjeux contemporains centraux en matière de RSE.

Ainsi, un des traits remarquables de la diffusion contemporaine de la RSE est l'émergence d'un ensemble de « marchés de la vertu ${ }^{10}$ (Vogel, 2005), d'une architecture de marchés, de standards et de nouvelles figures d'acteurs (consultants, certificateurs, agences de notation, de communication, investisseurs, gestionnaires de fonds, nouvelles fonctions dans les entreprises, etc.) (Acquier et Aggeri, 2007). Comme le souligne Gendron et al. (2004), ces marchés constituent des démarches de régulation d'une nature nouvelle, ne passant pas par les relais politiques traditionnels mais s'inscrivant directement dans le jeu économique. Se pose alors la question du potentiel de telles démarches en tant qu'outil de régulation économique (Capron et Quairel, 2004) et du rôle de la force publique dans

10. Par cette appellation, Vogel fait ici référence aux marchés de l'Investissement Socialement Responsable (Déjean, 2005), au commerce équitable et à d'autres produits marchands associés au développement durable. 
de telles démarches. Il apparaît difficile d'aborder de telles questions, pourtant essentielles, à travers la seule théorie des parties prenantes.

\section{L'absence de prise en compte des dynamiques de savoir et d'innovation}

Une troisième critique des approches stakeholders porte sur leur tropisme relationnel. Ainsi, ces approches visent avant tout à caractériser les responsabilités et les types de relations de l'entreprise vis-à-vis de ses parties prenantes, ainsi que leur prise en compte dans les processus de décision. Ces analyses s'opèrent le plus souvent dans un cadre statique, à ressources et périmètre constants. La plupart de ces approches s'inscrivent ainsi dans un paradigme du contrôle externe et de la contrainte (ou des devoirs de l'entreprise vis-à-vis de son environnement). Centrées sur la dimension relationnelle, elles ignorent une autre variable essentielle de l'action collective: les dynamiques de savoir et d'innovation (voir Hatchuel, 2000). Or l'examen des pratiques contemporaines d'entreprise en matière de responsabilité sociale et de développement durable suggère que ces dynamiques sont essentielles à prendre en compte pour comprendre les processus de création de ressources et de potentiels de valeur de l'entreprise (voir Aggeri et al., 2005). Ces pratiques accompagnent des activités de conception ou de reconception des pratiques, des dispositifs institutionnels (construction de marchés, nouvelles formes de réglementations), de nouvelles formes de partenariats entre entreprises et ONG, un travail de reconstruction de la valeur des produits et des services pour les clients. Dans cette perspective dynamique, d'un point de vue institutionnel comme du point de vue des entreprises, la notion de RSE n'est pas donnée. Il est alors possible d'analyser les stratégies en matière de développement durable ou de RSE comme un double processus de conception, où les dynamiques d'apprentissage alimentent et sont structurées par les dynamiques de normalisation (voir Aggeri et al., 2005). Cette perspective doit cependant être approfondie, d'une part, en revenant sur les questions d'organisation soulevées dans les années 1970 par le courant responsiveness, d'autre part, en s'interrogeant sur les mécanismes de développement de ressources (Barney, 1991) et de capacités dynamiques (Teece et al., 1997) générées par les politiques et les pratiques de RSE.

\section{CONCLUSION : DÉPASSER L'OPPOSITION \\ ENTREPRISE/SOCIÉTÉ POUR RENOUVELER LA POSTURE GESTIONNAIRE}

Quels sont les enseignements de notre analyse? La lecture généalogique des travaux du champ académique Business and Society suggère des mouvements de balancier entre deux postures distinctes, qui coexistent aujourd'hui sans vraiment dialoguer $^{11}$. La première est centrée sur la figure de l'entreprise (Business) et de ses managers. Elle étudie la manière dont l'entre-

11. Ces mouvements de balancier se manifestent par des requalifications périodiques du champ de recherche: Business and Society, Social issues in management, Business in society, corporate citizenship, etc. 
prise interagit avec ses parties prenantes et développe des stratégies légitimes ou acceptables, cherche à mettre à jour un lien positif entre les démarches de RSE et la performance financière de l'entreprise, ou des lois prédictives du comportement des entreprises à l'égard de ses parties prenantes externes. Cette littérature a souvent été critiquée pour l'ambiguïté de son projet: s'agit-il réellement d'améliorer l'inscription de l'entreprise dans la société ou de lui apprendre à déjouer les contre-pouvoirs qui l'entourent et ainsi de faciliter l'atteinte de ses objectifs, fussent-ils illégitimes? Soulignant les risques d'embrasser sans retenue l'intérêt managérial, une deuxième posture vise à juger de ces initiatives à l'aune de la société (society). En contrepoint des approches managériales, il s'agit d'évaluer l'impact des démarches d'entreprise sur l'intérêt social, le potentiel d'autorégulation des entreprises et les modalités de régulation juridiques des entreprises pour garantir une responsabilité sociale des entreprises effective. On peut aussi ranger dans cette catégorie les travaux centrés sur la gouvernance d'entreprise. De même, à un niveau plus local, le champ de l'éthique d'entreprise cherche à asseoir l'action managériale sur des fondements moraux souvent universels, issus de la philosophie morale. Cette seconde perspective n'est pas non plus exempte de critiques: en adoptant un point de vue plus externe et distancié de l'entreprise et des objectifs managériaux se pose la question de l'identité et du statut des travaux qui sont produits. En effet, en décentrant l'analyse de l'entreprise, ne sort-on pas d'un projet gestionnaire pour entrer dans les domaines de la sociologie, de l'éthique ou de l'économie? Quelle est la légitimité des gestionnaires, traditionnellement centrés sur les rationalisations de l'entreprise, à sortir de leur objet d'analyse?

Comme l'indique le tableau 1, il est possible de représenter l'hétérogénéité des différentes approches suivant deux axes: le point de vue adopté par le chercheur (business ou society) et son unité d'analyse (le manager, l'entreprise, ou la société). Cependant, on peut se demander si ces lignes de démarcation, construites au fil du temps, restent pertinentes pour analyser les dynamiques contemporaines en matière de RSE et de développement durable. Cellesci débordent en effet largement du cadre de l'entreprise et mettent simultanément en défaut les mécanismes traditionnels de l'action publique et de l'action managériale. Dès lors, comment dépasser la dichotomie entre entreprise et société ? À cette aune, il nous semble nécessaire d'adopter une définition élargie de la gestion consistant en l'étude des modes de pilotage de l'action collective, de leurs rationalisations et de leur réinvention permanente. Selon cette approche, la RSE ou le développement durable pose des questions fondamentales à la gestion, en interrogeant les frontières de l'entreprise, en accompagnant l'élaboration de nouveaux outils et métriques visant à internaliser les externalités, en interrogeant les modèles de création de valeurs associés aux pratiques de RSE et l'émergence de nouveaux modes de coordination entre acteurs. À cette aune, les questions de RSE ne constituent pas un champ autonome mais sont le traceur d'une rediscussion permanente des frontières entre action publique et privée et d'une extension progressive du périmètre de la gestion. 
Tableau 1 - Les différentes approches de la RSE, un essai de typologie

\begin{tabular}{|c|c|c|}
\hline Point de vue & & \\
\hline Unité d'analyse & Entreprise & Société \\
\hline Individu/Manager & $\begin{array}{l}\text { Corporate Social Responsiveness, } \\
\text { outils managériaux de déploiement } \\
\text { de la RSE (reporting, etc.) }\end{array}$ & $\begin{array}{l}\text { Éthique des affaires (fondements } \\
\text { moraux de l'action managériale) }\end{array}$ \\
\hline Entreprise & $\begin{array}{l}\text { Gestion des stakeholders, stratégies } \\
\text { politiques des entreprises, lien } \\
\text { performance sociétale/financière. }\end{array}$ & Gouvernance des entreprises \\
\hline Économie et société & $\begin{array}{l}\text { Corporate citizenship, pratiques de } \\
\text { stakeholder engagement }\end{array}$ & $\begin{array}{l}\text { Nouveaux indicateurs de mesure de } \\
\text { la richesse, école de la régulation et } \\
\text { potentiel de régulation de la RSE, } \\
\text { démocratie technique. }\end{array}$ \\
\hline
\end{tabular}

\section{Bibliographie}

Abrams F.W., "Management Responsibilities in a Complex World", Harvard Business Review, XXIX, 3 May 1951.

Abrahamson E., "Managerial fads and fashions: the diffusion and rejection of innovation", Academy of Management Review, vol. 16, $\mathrm{n}^{\circ} 3,1991$, p. 586-612.

Ackerman R. W., "How companies respond to social demand", Harvard Business Review, July-August 1973, p. 88-98.

Ackerman R. W., The Social Challenge to Busines, Cambridge, Massachusetts and London, England, Harvard University Press, 1975.

Ackerman R. W., Bauer R. A., Corporate Social Responsiveness - The Modern Dilemna, Reston Virginia, 1976.

Acquier A., Aggeri F., "Designing an industry: issues and theoretical frame. The case of the CSR reporting industry", Managing Corporate Social Responsibility in Action: Talking. Doing and Measuring, den Hond F., Bakker F. de, Neergaard P. (ed), Ashgate Publishing Group (à paraître), 2007.

Acquier A., Gond J.-P., « Aux sources de la Responsabilité Sociale de l'Entreprise: (re)lecture et analyse d'un ouvrage séminal: Social Responsibilities of the Businessman d'Howard Bowen (1953) », Communication à la conférence de l'AIMS, Angers, juin 2005.

Acquier A., Gond J.-P., "Repenser les théories quand les pratiques les transforment: les enjeux de la marchandisation de la Responsabilité Sociale de l'Entreprise ", Revue gestion, vol. $31, \mathrm{n}^{\circ} 2,2006 \mathrm{~b}$. 
Aggeri F., Acquier A., « La théorie des stakeholders permet-elle de rendre compte des pratiques d'entreprise en matière de développement durable ? ", Communication à la conférence de l'AIMS, Angers, juin 2005.

Aggeri F., Pezet E., Abrassart C., Acquier A., Organiser le développement durable, Paris, Vuibert, 2005.

Allouche J., Laroche P., "A meta-analytical examination of the link between corporate social and financial performance", Paper presented at the Academy of Management, Honolulu, Hawaï, 2005.

Andriof J., Waddock S., Husted B., Rahman S. S., Unfolding Stakeholder Thinking, vol. 1: Theory, Responsibility and Engagement, Sheffield, UK, Greenleaf Publishing, 2002.

Andriof J., Waddock S., Husted B., Sutherland Rahman S., Unfolding Stakeholder Thinking, Vol. 2: Relationships, Communication, Reporting and Performance, Sheffield, UK, Greenleaf Publishing, 2003.

Ansoff I., Corporate Strategy, New York, McGraw Hill, 1965.

Barney Jay B., "Firm Resources and Sustained Competitive Advantage", Journal of Management, vol. 17, $\mathrm{n}^{\circ} 1,1991$, p. 99-120.

Bauer R. A., Fenn D. H., The corporate Social Audit, New York, Russel Sage Foundation, 1972.

Bonnafous-Boucher M., Pesqueux Y. (Ed), Décider avec les parties prenantes. Approche d'une nouvelle théorie de la société civile, Éditions de La Découverte, 2006.

Bowen H. R., Social Responsibilities of the businessman, New York, Harper \& Brothers, 1953.

Burrell G., Morgan G., Sociological Paradigms and Organizational Analysis, Londres, Heinemann, 1979.

Calkins R. D., "Objectives of Business Education", Harvard Business Review, October 1946, p. 46-54.

Callon M., Lascoumes P., Barthes Y., Agir dans un monde incertain : Essai sur la démocratie technique, Paris, Le Seuil, 2001.

Capron M., Quairel-Lanoizelée F., Mythes et réalités de l'entreprise responsable. Acteurs, enjeux, stratégies, Paris, La Découverte, 2004.

Carroll A. B., "A Three Dimensional Conceptual Model of Corporate Performance", Academy of Management Review, vol. 4, n 4, 1979, p. 497-505.

Carroll A. B., "The Pyramid of Corporate Social Responsibility: Toward the Moral Managemen of Organizational Stakeholders", Business Horizons, July-August 1991, p. 39-48.

Carroll A. B., "Corporate social responsibility. Evolution of a definitional construct", Business \& Society, 38, 1999, p. 268-295.

Carson R., Silent Spring, Boston, Houghton Mifflin, 1962.

Charan R., Freeman R.E., "Stakeholders negociations: Building bridges with corporate constituents", Management Review, November 1979, p. 8-13.

Clark J. M., "The Changing Basis of Economic Responsibility", The Journal of Political Economy, vol. 24, n 3, 1916, p. 209-229. 
Clark J. M., Social Control of Business, 2nd ed., New-York, McGraw-Hill, 1939.

Clarkson M. B. E., "A stakeholder framework for analysing and evaluating Corporate Social Performance", Academy of Management Review, vol. 20, n 1, 1995, p. 92-117.

Cochran P. L., Wood D. J., "Corporate social responsibility and financial performance", Academy of Management Journal, 27, 1985.

Davis K., "Can business afford to ignore social responsibilities?", California Management Review, vol. 2, n 3, 1960.

Davis K., "The Case for and Against Business Assumptions of Social Responsibilities", Academy of Management Journal, vol. 16, $\mathrm{n}^{\circ} 2$, 1973, p. 312-322.

Déjean F., L'investissement socialement responsable. Étude du cas français, Vuibert, 2005.

Déjean F., Gond J.-P. et Leca B., "Measuring the unmeasured: an institutional entrepreneur strategy in an emerging industry", Human Relations, vol. 57, $n^{\circ}$ 6, 2004, p. 741-764.

Dierkes M., Bauer R. A., Corporate Social Accounting, New York, Praeger Publishers, 1974.

Dierkes M., Berthoin Antal A.,"Whither corporate social reporting: Is it time to legislate?", California Management Review, vol. 28, n 3, 1986.

Donaldson T., Preston L. E., "The Stakeholder Theory of the Corporation: Concepts, Evidence, and Implications", Academy of Management Review, vol. 1, $\mathrm{n}^{\circ}$ 20, 1995, p. 65-91.

Donham W. B., "The Emerging Profession of Business", Harvard Business Review, vol. 5, $n^{\circ} 4,1927$.

Drucker P. F., The practice of management, Paris (version française 1957), Éditions d'Organisation, 1954.

Elkington J., Cannibals With Forks. The tripple bottom line of the $21^{\text {st }}$ century business, Oxford, Capstone publishing, 1998.

Emshoff J. R., Freeman R. E., "Stakeholder Management", Working paper, Wharton Applied Research Center, 1978.

Epstein E. M., "The Field of Business Ethics in the United States: Past, Present and Future", Journal of General Management, vol. 28, $\mathrm{n}^{\circ} 2,2002$, p. 1-29.

Frederick W. C., "Coda", Business and Society, vol. 33, n 2, 1994.

Freeman R. E., Reed D. L., "Stockholders and Stakeholders: A New Perspective on Corporate Governance", California Management Review, vol. 25, n 3, 1983, p. 88-107.

Freeman R. E., Strategic management : A stakeholder approach, Marshfield, Massachussets, Pitman Publishing, Inc, 1984.

Freeman R. E., Evan W. M., “Corporate Governance: A stakeholder interpretation”, Journal of Behavioral Economics, vol. 19, n 4, 1990.

Freeman R. E., "The politics of stakeholder theory: some future directions", Business Ethics Quarterly, vol. 4, n 4, 1994, p. 409-422.

Freeman, R. E., "Divergent Stakeholder Theory", Academy of Management Review, vol. 24, $n^{\circ} 2,1999$, p. 233-236.

Friedman M., Capitalism and Freedom, Chicago University Press, 1962.

Frooman J., "Stakeholder Influence Strategies", Academy of Management Review, vol. 24, $\mathrm{n}^{\circ} 2,1999$, p. 191-205. 
Garriga E., Melé D., "Corporate Social Responsibility Theories: Mapping the Territory", Journal of Business Ethics, vol. 53, $\mathrm{n}^{\circ} 2,2004$, p. 51-71

Galbraith J. K., Le nouvel état industriel, Gallimard, 1969.

Gendron C., Lapointe A. et Turcotte M.-F., « Responsabilité sociale et régulation de l'entreprise mondialisée », Relations industrielles/industrial relations, vol. 59, $\mathrm{n}^{\circ}$ 1, 2004, p. $71-100$.

Hatchuel A., "Quel horizon pour les sciences de gestion ? Vers une théorie de l'action collective ", Les nouvelles fondations des sciences de gestion, éléments d'épistémologie en management, David A., Hatchuel A. and Laufer R. (Eds). Paris, Vuibert, 2000.

Hatchuel A., Pezet E., Starkey K. et Lenay O. (Eds), Gouvernement, organisation et gestion : l'héritage de Michel Foucault, Presses Universitaires de Laval, 2005.

Heald M., "Business Thought in the Twenties: Social Responsibility", American Quarterly, vol. 13, n², 1961, p. 126-139.

Heald M., The Social Responsibilities of Business: Company and Community, 1900-1960, Cleveland, Case Western Reserve University Press, 1970.

Jarniou P., L'entreprise comme système politique, Paris, PUF, 1981.

Jawahar I. M., Mclaughlin G. L., "Toward a descriptive stakeholder theory: an organizational life cycle approach", Academy of Management Review, vol. 26, n 3, July 2001.

Jensen M.C., Meckling W. H., "Theory of the Firm: Managerial Behavior, Agency Costs and Ownership Structure", Journal of Financial Economics, vol. 3, n 4, 1976, p. 305-360.

Jones T. M., "Corporate Social Responsibility Revisited, Redefined", California Management Review, XXII (2), 1980.

Jones T. M., "Instrumental stakeholder theory: a synthesis of ethics and economics", Academy of Management Review, vol. 20, $\mathrm{n}^{\circ} 2,1995$, p. 404-437.

Laufer R., "Quand diriger, c'est légitimer », Revue française de gestion, novembredécembre 1996.

Malott D. W., "Business Advancing as a Profession", Iron Trade Review, June 12, 1924, p. 1564-1565.

Management Review, "How AT\&T copes with its stakeholders", vol. 69, n 8, 1980, p. 4-8.

Margolis J. D., Walsh J. P., "Misery Loves Companies : Rethinking Social Initiatives by Business", Administrative Science Quarterly, 2003.

Martinet A.C., Management stratégique: organisation et politique, Mc Graw Hill, 1984.

Martinet A.-C., Reynaud E., Management stratégique et écologie, Economica, 2004.

Mitchell R. K., Agle B. R., Wood D. J., "Toward a theory of stakeholder identification and salience : defining the principle of who and what really counts", Academy of Management Review, vol. 22, $\mathrm{n}^{\circ} 4,1997$, p. 853-886.

Mitchell R. K., Agle B. R., Sonnenfield J. A., "Who matter to CEO? An investigation of Stakeholders Attributes and Salience, Corporate Performance, and CEO values", Academy of Management Journal, vol. 42, n 5, 1999. 
Moon J., Crane A., Matten D., "Can Corporations Be Citizens? Corporate Citizenship as a Metaphor for Business Participation in Society", Business Ethics Quarterly, vol. 15, $\mathrm{n}^{\circ} 3$, 2005, p. 429-453.

Moskotitz M. R., "Choosing Socially Responsible Stocks", Business and Society Review, $\mathrm{n}^{\circ} 1,1972$, p. $72-75$.

Nader R., Unsafe at Any Speed, Pocket Books, 1965.

Norman W., MacDonald C., "Getting to the bottom line of the tripple bottom line", Business Ethics Quarterly, vol. 14, n², 2004, p. 243-263.

Orlitzky M., Schmidt F. L. et Rynes S. L., "Corporate social and financial performance:A meta-analysis", Organization Studies, vol. 24, n 3, 2003, p. 103-141.

Pasquero J., « L'entreprise face aux pressions socio-politiques de son environnement », document de travail $\mathrm{n}^{\circ} 80-100$, IAE-IEC Grenoble, juin 1980.

Pasquero J., «La responsabilité sociale de l'entreprise comme objet des sciences de gestion: Un regard historique ", Responsabilité sociale et environnementale de l'entreprise, Turcotte M.-F. et Salmon A. (Eds.), Presses de l'Université du Québec, 2005.

Pfeffer J., Salancik G. R., The external control of organizations: a ressource dependance perspective, Harper et Row Publishers, 1978.

Phillips R., Stakeholder theory and organizational ethics, San Francisco, Berrett-Koehler, 2003.

Phillips R., Freeman R. E., Wicks A. C., "What Stakeholder Theory Is Not", Business Ethics Quarterly, vol. 13, n 4, 2003, p. 479-502.

Post J., Preston L. E. et Sauter-Sachs S., Redefining the Corporation: Stakeholder Management and Organizational Wealth, Stanford University Press, 2003.

Prahalad C. K., Hart S. L., "The fortune at the bottom of the pyramid", Strategy and Business, 26, 2002, p. 1-14.

Rowley T. J., “Moving Beyond Dyadic Ties: A Network Theory of Stakeholder Influences", Academy of Management Review, vol. 22, $\mathrm{n}^{\circ} 4,1997$, p. 887-910.

Rowley T. J., Moldoveanu M., "When will stakeholder groups act? An interest- and identitybased model of stakeholder group mobilization", Academy of Management Review, vol. $28, n^{\circ} 2,2003$, p. $204-219$.

Scholte A., "Globalization, governance and Corporate Citizenship", Journal of Corporate Citizenship, 1, 2001, p. 15-23.

Sturdivant F. D., “Executives and Activists: Test of stakeholder Management", California Management Review, XXII (1), 1979.

Swanson D. L., "Adressing a theoretical problem by reorienting the corporate social performance model", Academy of Management Review, vol. 20, n 1, 1995, p. 43-64.

Swanson D. L., "Toward an integrative theory of business and society: a research strategy for corporate social performance", Academy of Management Review, vol. 24, n 3, 1991, p. 506-521.

Teece D. J., Pisano G. et al., "Dynamic Capabilities and Strategic Management", Strategic Management Journal, vol. 18, 1997, p. 509-533. 
Trevino L. K., Weaver G., "The Stakeholder Research Tradition: Converging Theorists - Not Converging Theory", Academy of Management Review, vol. 24, $\mathrm{n}^{\circ}$ 2, 1999, p. 222-227.

Vogel D., "The Study of Social Issues in Management: A Critical Appraisal", California Management Review, XXVIII 2, Winter, 1986.

Vogel D., The Market for Virtue - The Potential and Limits of Corporate Social Responsibility, Washington, Brookings Institution Press, 2005.

Waddock S., Graves S. B., "Quality of Management and Quality of Stakeholders Relations: Are they Synonymous?", Business \& Society, 105, 1997a, p. 47-62.

Waddock S., Graves S. B., "The Corporate Social Performance - Financial Performance Link", Strategic Management Journal, vol. 18, n 4, 1997b, p. 313-319.

Walsh J. P., "Book Review Essay: Taking Stock of Stakeholder Management", Academy of Management Review, vol. 30, $\mathrm{n}^{\circ} 2,2005$, p. 426-438.

Wartick S. L., Cochran P. L., "The Evolution of the Corporate Social Performance Model", Academy of Management Review, vol. 10, $\mathrm{n}^{\circ} 4,1985$.

Wartick S. L., Rude R. E., "Issues Management: Corporate Fad or Corporate Function?", California Management Review, XXIX 1, Fall, 1986.

WBCSD, The Business Case for Sustainable Development, Switzerland, WBCSD, 2002.

Weaver G., Trevino L. K., Cochran P. L., "Integrated and Decoupled Corporate Social Performance: Management Commitments, External Pressures, and Corporate Ethics Practices", Academy of Management Journal, vol. 42, n 5, 1999, p. 539-552.

Wood D. J., "Social Issues in Management: Theory and Research in Corporate Social Performance", Journal of Management, vol. $17 \mathrm{n}^{\circ}$ 2, 1991a, p. 383-406.

Wood D. J., "Corporate Social Performance Revisited", Academy of Management Review, vol. 16, $n^{\circ} 4,1991 b$.

Wood D. J., "Theory and Integrity in Business and Society", Business \& Society, vol. 39, $\mathrm{n}^{\circ} 4,2000$, p. 359-378.

Zadek S., "The Path to Corporate Responsibility", Harvard Business Review, décembre 2004. 


\section{ONT CONTRIBUÉ À CE NUMÉRO}

Aurélien ACQUIER est professeur assistant à l'ESCP-EAP et chercheur associé au CGS de l'École des mines de Paris. Il enseigne la stratégie, les théories des organisations et le management du développement durable. Il a récemment soutenu sa thèse, intitulée «Les modèles de pilotage du développement durable: du contrôle externe à la conception innovante».

Franck AGGERI est maître-assistant à l'École des mines de Paris où il dirige le Centre de gestion scientifique (CGS). Ses recherches portent sur la stratégie, le développement durable et le management de l'environnement. Il a publié en 2005 chez Vuibert Organiser le développement durable: expériences des entreprises pionnières et formation de règles d'action collective (en collaboration avec É. Pezet, C. Abrassart et A. Acquier) et a coordonné avec Olivier Godard un numéro spécial de la revue Entreprises et Histoire sur les « Entreprises et le développement durable » (2006).

Mina BÉCHEUR est maître de conférences à l'université Paris-Est, Marne-laVallée. Ses travaux portent sur l'émergence et la diffusion de nouveaux concepts, avec un focus plus particulier sur le commerce équitable et la responsabilité sociale de l'entreprise dans ses derniers travaux.

Faouzi BENSEBAA est maître de conférences habilité à diriger les recherches à l'université Paris 12. Il enseigne la stratégie et la responsabilité sociale, codirige le département de management de I'IRG et conduit ses recherches sur la responsabilité sociale de l'entreprise et la dynamique concurrentielle. Il vient de publier L'atlas $d u$ management (en collaboration avec D. Autissier et F. Boudier, Ed. Eyrolles).

Jean-Pierre BOISSIN est professeur à l'université Pierre Mendès France (IAE de Grenoble) en management stratégique et entrepreneuriat. Il a fondé et dirigé la Maison de l'entrepreneuriat de Grenoble universités, projet pilote pour le ministère de la Recherche. Il est membre de différentes associations académiques et participe à plusieurs comités d'évaluation de revues. Il est depuis 2006 le directeur du Centre d'études et de recherches appliquées à la gestion (CERAG, université Pierre Mendès France). Ses travaux de recherche portent sur la structuration du management stratégique à partir d'outils bibliométriques. Il développe actuellement un observatoire international de l'intention entrepreneuriale et dirige différents travaux sur les stratégies de croissance et le gouvernement d'entreprise.

Marc BOLLECKER, docteur en sciences de Gestion, est maître de conférences à l'université de Haute Alsace. Il est membre du Groupe de recherches sur l'apprentissage, l'innovation et la connaissance dans les organisations (GRAICO) de 